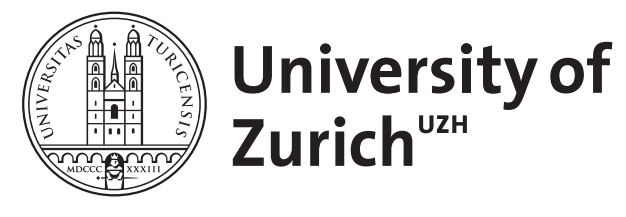

\title{
The evolution of sex roles in mate searching
}

\author{
Fromhage, Lutz ; Jennions, Michael ; Kokko, Hanna
}

\begin{abstract}
Searching for mates is a critical stage in the life cycle of most internally, and many externally, fertilizing species. Males usually invest more in this costly activity than females, but the reasons for this are poorly understood. Previous models have shown that female-biased parental investment, including anisogamy, does not by itself select for male-biased mate searching, so it requires additional explanations. Here, we correct and expand upon earlier models, and present two novel hypotheses that might explain the evolution of male-biased mate searching. The "carry-over hypothesis" states that females benefit less from searching if the associated costs affect other stages of the life cycle, rather than arising only while searching. It is relevant to the evolution of morphological traits that improve searching efficiency but are also expressed in other contexts. The "mating window hypothesis" states that females benefit less from searching if their life cycle includes intervals during which the exact timing of mating does not matter for the appropriate timing of reproduction (e.g., due to sperm storage or delayed embryo implantation). Such intervals are more likely to exist for females given the general pattern of greater female parental investment. Our models shed new light on classic arguments about sex role evolution.
\end{abstract}

DOI: https://doi.org/10.1111/evo.12874

Posted at the Zurich Open Repository and Archive, University of Zurich

ZORA URL: https://doi.org/10.5167/uzh-137408

Journal Article

Accepted Version

Originally published at:

Fromhage, Lutz; Jennions, Michael; Kokko, Hanna (2016). The evolution of sex roles in mate searching. Evolution, 70(3):617-624.

DOI: https://doi.org/10.1111/evo.12874 


\section{The evolution of sex roles in mate-searching}

\section{Running title: Evolution of mate-searching}

Lutz Fromhage $^{1 *}$, Michael Jennions ${ }^{2}$ \& Hanna Kokko ${ }^{3}$

${ }^{1}$ Department of Biological and Environmental Science, University of Jyvaskyla, P.O. Box 35, 40014 Jyvaskyla, Finland

${ }^{2}$ Ecology, Evolution \& Genetics, Research School of Biology, Australian National University, Canberra ACT 0200, Australia

${ }^{3}$ Institute of Evolutionary Biology and Environmental Studies, University of Zurich, Winterthurerstrasse 190, $\mathrm{CH}-8057$ Zurich, Switzerland

*Corresponding author

E-mail addresses:

lutzfromhage@web.de

Michael.Jennions@anu.edu.au

hanna.kokko@ieu.uzh.ch

Key words: Parental investment, mating effort, sex roles, models/simulations.

Word count: 4364

Table count: 0

Figure count: 4 
Abstract

2 Searching for mates is a critical stage in the life-cycle of most internally, and many externally,

3 fertilising species. Males usually invest more in this costly activity than females, but the reasons

4 for this are poorly understood. Previous models have shown that female-biased parental

5 investment, including anisogamy, does not by itself select for male-biased mate searching, so it

6 requires additional explanations. Here we correct and expand upon earlier models, and present

7 two novel hypotheses that might explain the evolution of male-biased mate searching. The 'carry-

8 over hypothesis' states that females benefit less from searching if the associated costs affect other

9 stages of the life-cycle, rather than arising only while searching. It is relevant to the evolution of

10 morphological traits that improve searching efficiency but are also expressed in other contexts.

11 The 'mating window hypothesis' states that females benefit less from searching if their life-cycle

12 includes intervals during which the exact timing of mating does not matter for the appropriate

13 timing of reproduction (e.g. due to sperm storage or delayed embryo implantation). Such intervals

14 are more likely to exist for females given the general pattern of greater female parental

15 investment. Our models shed new light on classic arguments about sex role evolution.

Introduction

18 Searching for mates is a critical stage in the life-cycle of most internally, and many externally,

19 fertilising species. In a broad sense, search effort can be defined as a costly investment in traits

20 that facilitate encounters with potential mates; including mobility, advertisement calls or displays,

21 and pheromone production (Kokko and Wong 2007). Often such traits are sexually dimorphic, with

22 males typically exhibiting higher levels of effort (Andersson 1994; Birkhead and Moller 1998;

23 Simmons 2001). A classic explanation for this asymmetry is that, in species where females have

24 lower potential reproductive rates than males driven by their greater parental investment, males 
benefit more than females from elevating their mating rate (Bateman 1948; Trivers 1972; CluttonBrock and Vincent 1991). On closer inspection, however, the simplicity of this argument is deceptive: in a formal model of the evolution of mate-searching, Hammerstein and Parker (1987) found that, regardless of asymmetry in parental investment, there exist alternative evolutionarily stable strategies (ESSs) of male-only or female-only searching. On the other hand, they noted that "the selective forces which stabilise a high level of male mobility are stronger than those which stabilise a high level of female mobility", concluding that it would be desirable to determine the 'range of attraction' of each ESS in an explicitly dynamic model of the selection process. Taking up this challenge, Kokko and Wong (2007) presented a model which described such ranges of attraction, reporting that these are symmetrical when the sexes differ only in their extent of parental investment. As we will show, however, this conclusion was incorrect. Our present paper therefore supersedes Kokko and Wong (2007), which contained technical errors that have led to its retraction (see Appendix).

Here we derive a new baseline model for the evolution of mate searching where the sexes only differ in their level of parental investment. We then explore two extensions of the model based on consequences of anisogamy that have largely been overlooked, but are likely to be of general importance. These are respectively related to the existence of 'mating windows' and of 'carry over' effects (both defined below).

In species with internal fertilisation, females' life cycle typically includes intervals we will call 'mating windows', during which the exact timing of mating does not affect the timing of offspring production, so long as sperm is acquired before zygotes need to develop. Depending on the species, mating windows can be short (e.g., a few hours around ovulation) or long (e.g. weeks or 
months in organisms with specialized sperm storage organs; Orr and Brennan 2015). According to our definition, a mating window begins when a female is ready to receive sperm for the fertilisation of her next (batch of) offspring, and it ends at the latest insemination point that would ensure reproduction at the earliest (optimal) time permitted by other intrinsic and extrinsic factors, without unnecessary delay due to lack of sperm. For example, certain forms of parental investment (e.g., formation of nutrient-rich eggs, or building up reserves for pregnancy) take time before reproduction. Similarly, females must sometimes wait for suitable external conditions to breed. If females schedule their mating activity to overlap with such delays, a mating window arises that allows them to acquire sperm before it is needed. The intervening time can be bridged by mechanisms such as sperm storage or embryonic diapause. An important consequence of this is that, if females can expect to mate at least once per mating window, they should be in no hurry to acquire a mate. If they mate earlier they will just have to wait longer afterwards, until the mating window ends, which confers no benefit. By contrast, a male only has to wait until he has replenished his sperm supply before being ready to reproduce again (with a different female). A male that mates sooner will therefore return to the mating pool sooner and this, all else being equal, should elevate his lifetime mating success. A mating window presumably weakens any female incentive to invest in mate searching, for a reason that is logically distinct from the extent of parental investment per se. Mating windows do not require that females mate multiply, so their occurrence is conceptually distinct from that of polyandry and sperm competition. We make this point because previous models have explicitly included mating windows to generate polyandry (e.g. Kokko \& Wong 2007). Here we show how the existence of mating windows for females, independent of any role for polyandry, affects the evolution of sex roles for mate searching. 
Previous models of mate searching have constrained the mortality costs of mate searching to those that arise while searching. It is, however, plausible that investment into searching more efficiently, or more intensely, will have 'carry over' mortality costs that affect other stages of the life-cycle. This is obviously relevant to the evolution of morphological traits that are not at their naturally selected optimum because this improves searching efficiency, but are then expressed in non-searching contexts where they might be disfavoured (e.g. body size, energetically costly musculature). More generally, it is possible that investment into mate searching reduces other fitness components (e.g. lowers fecundity). 'Carry-over' effects are likely to be important because previous models indicate that general mortality costs of sexually selected traits can create a sexual asymmetry in the scope of investment into traits that elevate mating rates (Kokko et al. 2012).

\section{Model framework}

Following a tradition in the literature of sex role evolution (e.g. Clutton-Brock and Parker 1992; Kokko and Monaghan 2001), we distinguish two life phases during which individuals are either available for mating and 'in the mating pool' ('time-in') or unavailable ('time-out'). Time-out arises while processing the consequences of mating (fig 1a-c) or, in one model variant (fig. 1b), while waiting for the end of a mating window. If time-out is used to accumulate resources to be invested in offspring, its duration can be interpreted as a measure of parental investment (Trivers 1972). Adult life begins in the 'time-in' state, and individuals alternate between states according to constant transition rates. We used a continuous time model where events are asynchronous among individuals, leading to stable state frequencies. For simplicity, we assume that every encounter between individuals in the 'time-in' state results in mating. 
98

99

$$
Q=\left(\begin{array}{cccc}
-\mu_{O}-\frac{1}{T} & m & 0 & 0 \\
\frac{1}{T} & (g-1) m-\mu_{I} & 0 & g \tilde{m} \\
0 & 0 & -\tilde{\mu}_{O}-\frac{1}{\tilde{T}} & \tilde{m} \\
0 & g m & \frac{1}{\tilde{T}} & (g-1) \tilde{m}-\tilde{\mu}_{I}
\end{array}\right)
$$

Here, element $q_{\mathrm{i}, \mathrm{j}}$ (in row $\mathrm{i}$, column $\mathrm{j}$ ) specifies the per-capita rate at which individuals of state $\mathrm{j}$ contribute to entering and leaving of state i (see Hardling et al. 2003). The relevant states are (from left to right, and top to bottom): females in time-out, females in time-in, males in time-out, males in time-in. For example, the first entry $q_{1,1}$ states that females in time-out disappear at rate $-\mu_{O}$ owing to mortality, and at rate $-1 / T$ owing to their return to the mating pool, where they re-appear at rate $1 / T$ in element $q_{2,1}$. So $T$ is the average duration of female time-out. (The

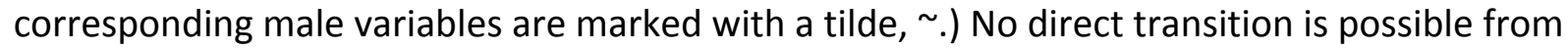
females in time-out to males of any kind, so the last two entries in this column are zeros. In the second column, element $q_{1,2}$ states that females transition from time-in to time-out at their mating rate, $m$, implying that females mate only once per reproductive cycle. Mating leads to the production of $2 g$ adult offspring of each sex, which enter time-in in elements $q_{2,2}, q_{2,4}, q_{4,2}$, and $q_{4,4}$, respectively. This corresponds to a (genetic) contribution of $g$ per parent, where $g$ is chosen such that population size remains stable. Offspring are produced immediately upon mating (but see mating window model below). Females in time-in are subject to mortality $\mu_{I}$. Throughout, we assume an even primary sex ratio. Defining the operational sex ratio $\beta$ as the ratio between males and females in time-in (derived by eq. 3. in Kokko and Monaghan 2001), we let mating rates be 
$117 m[x, \beta]=M f[x, y] \sqrt{\beta}$ and $\widetilde{m}[y, \beta]=M f[x, y] \frac{1}{\sqrt{\beta}}$, to satisfy the consistency requirement that 118 they must be linked as $m / \widetilde{m}=\beta$. (Here and elsewhere, we omit function arguments for brevity.)

119 The 'encounter coefficient' $M$ captures species-specific factors such as movement efficiency and 120 population density, and the 'search function' $f[x, y]$ specifies how female effort $(x)$ and male 121 effort $(y)$ jointly affect the search outcome. We use two forms of this function: the additive case $122 f[x, y]=x+y$ describes situations where sufficient search effort in one sex may remove the 123 need to search in the other sex. The multiplicative case $f[x, y]=x y$ describes situations where 124 both sexes must invest some effort in order to meet. To control how fast mortality increases with 125 search effort, we define a search cost coefficient $c$ such that $\mu_{I}[x]=0.1\left(1+x^{c}\right)$ and $\tilde{\mu}_{I}[y]=$ $1260.1\left(1+y^{c}\right)$.

127 We use two alternative methods to calculate selection gradients for this model, to improve our 128 confidence in the robustness of the analysis. First, we calculate the selection gradient of trait $k$ as 129 the partial derivative of the dominant eigenvalue $\lambda$ of the transition matrix with respect to a rare 130 mutant's $k$, as $\sum_{i, j} u_{j} v_{i} \frac{\partial q_{i, j}}{\partial k}$ (Caswell 1978; Pen and Weissing 2000), where $\mathbf{u}$ is the dominant right 131 eigenvector containing stable state frequencies, and $\mathbf{v}$ is the dominant left eigenvector containing reproductive values (normalised such that $\sum_{i} u_{i} v_{i}=1$ ). The eigenvectors and $g=\frac{\mu_{I}+m T \mu_{O}+T \mu_{I} \mu_{O}}{2 m\left(1+T \mu_{O}\right)}$ are obtained by solving the systems $\mathbf{v} \cdot \mathbf{Q}=0$ and $\mathbf{Q} \cdot \mathbf{u}=0$ (Hardling et al. 2003). If searching affects mortality only during time-in, then using the information that $u_{4} / u_{2}=\beta$ (by our definition of $\beta$ ) and $v_{2}=v_{4}$ (the so-called 'Fisher condition', which states that fitness, and hence reproductive value at birth, is equal between the sexes when the primary sex ratio is equal; 137 Houston and McNamara 2005) we obtain selection gradients (up to a constant): 
$141 \quad \frac{\partial \lambda}{\partial y}=\beta\left(\left(2 g-1+\frac{1}{1+\widetilde{\mu}_{O} \tilde{T}}\right) \widetilde{m}^{\prime}-\tilde{\mu}_{I}^{\prime}\right)$

143 These are the corrected versions of Kokko and Wong's (2007) eqns. 5, which failed to take into

144 account how the duration of a trait's expression affects its exposure to selection (see Appendix).

145 Second, we calculate selection gradients as proportional derivatives of lifetime reproductive

146 success (fitness) of a rare mutant of each sex, $\frac{\partial W[x, \beta]}{\partial x W[x, \beta]}$ and, $\frac{\partial \widetilde{W}[y, \beta]}{\partial y \widetilde{W}[y, \beta]}$. Although originally designed

147 for discrete-time models (McElreath and Boyd 2007, p 299), this method yields results for the

148 relative strength of selection that are identical to the previous method (both methods generate

149 figures 2-4). Importantly, however, the fitness function used in this method also allows us to find 150 ESSs analytically. Fitness (equivalent to the expected number of matings, hence breeding events)

151 of a mutant female with search effort $x$ is given by

152

$153 W[x, \beta]=p\left(1+\sum_{i=1}^{\infty}(s p)^{i}(1-s p) i\right)=\frac{p}{1-s p}$

154

155 where $p=\frac{m}{m+\mu_{I}}$ is the probability that a given search ends in mating (rather than death),

$156 s=\frac{1 / T}{1 / T+\mu_{O}}$ is the probability that a given time-out ends by returning to the mating pool (rather

157 than death), $s p$ is the probability that at least one more mating will follow after any given mating, 158 and $(s p)^{i}(1-s p)$ is the probability of mating exactly $i$ additional times.

\section{Mating window model}

161 Here we assume that, at the beginning of their reproductive life, and after every time-out, females 162 enter a mating window, defined as an interval during which the exact timing of mating does not 
matter for the timing (hence the fitness gain) of subsequent reproduction. If mating does not happen during a given mating window, the female immediately enters another mating window. Unmated females transition to a 'time-lag' state upon mating (fig. 1 b-c). Depending on parameter settings (see below), females can or can not re-mate during the time-lag. To capture the idea that females who mate early during a mating window of duration $F$ face a correspondingly longer time-

lag, we derive time-lag duration as:

$L[x, \beta]=\frac{\int_{0}^{F} m \cdot e^{-t\left(\mu_{I}+m\right)} \cdot(F-t) d t}{\int_{0}^{F} m \cdot e^{-t\left(\mu_{I}+m\right)} d t}=\frac{F\left(\mu_{I}+m\right)+e^{-F\left(\mu_{I}+m\right)}-1}{\left(\mu_{I}+m\right)\left(1-e^{-F\left(\mu_{I}+m\right)}\right)}$

where $m \cdot e^{-t\left(\mu_{I}+m\right)} d t$ is the probability that a female mates for the first time at $t$, in which case

171 she faces time-lag duration $F-t$; the term $e^{-t\left(\mu_{I}+m\right)}$ represents the probability that she has

172 neither mated nor died before $t$. To keep track of females during the time-lag, we introduce a fifth 173 state in the transition matrix, in the fifth row and column:

$Q=\left(\begin{array}{ccccc}-\mu_{O}-\frac{1}{T} & 0 & 0 & 0 & \frac{1}{L} \\ \frac{1}{T} & -m-\mu_{I} & 0 & \frac{g \rho \tilde{m}}{n} & \frac{g}{L} \\ 0 & 0 & -\tilde{\mu}_{O}-\frac{1}{\tilde{T}} & \tilde{m} & 0 \\ 0 & 0 & \frac{1}{\tilde{T}} & \left(\frac{g \rho}{n}-1\right) \tilde{m}-\tilde{\mu}_{I} & \frac{g}{L} \\ 0 & m & 0 & 0 & -\mu_{L}-\frac{1}{L}\end{array}\right)$

176 Here, $1 / L$ is the rate at which females transition from time-lag to the 'processing state' (now in

177 row and column one; see fig. 1). The term $\rho[x, \beta]=\frac{1 / L}{1 / L+\mu_{L}}$ is the probability of surviving a time-

178 lag, and $n[x, \beta]=1+\int_{0}^{\infty} e^{-t\left(\mu_{L}+\frac{1}{L}\right)} m_{L} d t=1+\frac{L m_{L}}{1+L \mu_{L}}$ is the expected number of matings per

179 female of those that survive to breed. Here, $\mu_{L}$ and $m_{L}$ are mortality and mating rate during the time-lag. In the monandry case, we substitute $\mu_{L}$ with $\mu_{\mathrm{O}}$ and $m_{\mathrm{L}}$ with 0 , implying that females 
spend the time-lag in time-out, so that $n=1$. In the polyandry case, for simplicity, we substitute $\mu_{L}$ with $\mu_{I}$ and $m_{\mathrm{L}}$ with $m$, implying that females spend the time-lag behaving like unmated, timein females. Offspring are produced during the transition from time-lag to processing state. In elements $q_{2,4}$ and $q_{4,4}$, males' reproductive success per mating is discounted by the probability $\rho$ that their mate survives to reproduce, and by average paternity $1 / n$. Female fitness is now $W[x, \beta]=\frac{p \rho}{1-s p \rho}$, and male fitness is $\widetilde{W}[y, \beta]=\frac{\tilde{p}}{1-\tilde{s} \tilde{p}} \cdot \frac{\rho}{n}$ where the factor $\rho / n$ accounts for shared paternity and female death before reproduction. Using the 'Fisher condition' requirement that average male and female fitness are linked by the primary sex ratio $r$, we calculate the operational sex ratio $\beta$ by numerically solving the equation $W[x, \beta]=\widetilde{W}[y, \beta] \cdot r$ for given resident strategies $x$ and $y$. We then proceed as before to calculate selection gradients on male and female search effort.

\section{Results}

\section{Basic model}

Depending on the search function $f$ and search cost coefficient $c$, we predict ESSs where both sexes invest equally in mate searching, or where either sex does all of the searching (fig. 2). The location of these equilibria is symmetrical, in the sense that for every equilibrium with unequal searching, there exists an equivalent equilibrium with reversed roles. Crucially, however, the corresponding trajectories (hence ranges of attraction) are not symmetrical. This is most notable if mate encounter rates are high (high $M$ ) because male effort then evolves much faster than female effort (vertical trajectories in bottom row of fig. 2), reflecting stronger selection on males. Where alternative ESSs exist (first panel of fig. 2), outcomes with male-biased searching are therefore more accessible from low-effort ancestral conditions (because males are quicker to evolve away

204 from maladaptively low levels of searching); outcomes with female-biased searching are more 
accessible from high-effort ancestral conditions (because males are quicker to evolve away from maladaptively high levels of searching). To derive ESS solutions analytically, we note that fitness is maximised when the probability $p=\frac{m}{m+\mu_{I}}$ of surviving a given time-in is maximised, provided that searching affects only mortality during time-in. Because $d p / d x$ is positive when increasing $x$ causes higher proportional changes in $m$ than in $\mu_{I}$, the requirement $\mathrm{d} p / \mathrm{d} x=0$ of an ESS with $x>$

2100 implies $\frac{d m}{d x \cdot m}=\frac{d \mu_{I}}{d x \cdot \mu_{I}}$. With our definition of $m$, this leads to the implicit solution

$211 \frac{d f}{d x \cdot f}=\frac{d \mu_{I}}{d x \cdot \mu_{I}}$, which illustrates three general properties of the ESS. It is: 1 ) independent of $\left.M ; 2\right)$ 212 independent of $\beta$, hence of parental investment as measured by time-out durations $T$ and $\widetilde{T} ; 3$ ) symmetric between the sexes. The equation depends only on $f$ and $\mu_{I}$, so it follows that the same equilibria apply to each sex if these functions are the same for each sex. For the multiplicative case $(f=x y)$, this simplifies to the closed-form solution: $x_{E S S}=y_{E S S}=(c-1)^{-1 / c}$.

The symmetry of ESSs can be broken in favour of male-biased searching if we assume there are search-related mortality costs that extend beyond the period of searching, as posited by our 'carry over' hypothesis (fig. 3a). These costs generate an asymmetry because mortality outside of the period of searching (i.e., during time-out) is disproportionately costly for the sex (here: females) that spends a greater proportion of its time in this state. The symmetry of ESSs can also be broken

221 if females pay higher mortality costs while searching than do males for the same amount of effort (fig. 3b).

Mating window model

225 This model yields ESSs with male-biased searching under both monandry and polyandry, and even if processing times $T$ and $\widetilde{T}$ are the same for both sexes (fig. 3c-d). The analytic argument outlined 
above still applies for males, so their ESS effort still depends only on $f$ and $\tilde{\mu}_{I}$. Females' ESS effort, however, now decreases as the mate encounter coefficient $M$ increases (fig. 4). This result holds for both search functions, but is easiest to visualize for the multiplicative case which has no alternative ESSs. Female search effort also decreases with mating window duration $F$ under broad 231 conditions (fig. 4).

\section{Discussion}

234 In nature there is a general trend for males to invest more than females in mate-searching. A 235 previous model by Hammerstein and Parker (1987) showed that this pattern is not attributable to greater female than male parental investment (including anisogamy itself) and the longer 'timeout' after mating of females. A subsequent model by Kokko and Wong (2007) stated that, in addition to the ESSs being symmetric, the strength of selection on each sex is also symmetric even if there are sex differences in parental investment; but that it is possible for male-biased searching

240 to evolve if females are polyandrous or if they pay a higher search cost than males (Kokko and 241 Wong 2007). Here we re-examine these claims and find that only the latter can be substantiated.

242 We then provide support for two additional explanations for the evolution of male-biased mate 243 searching.

\section{Three explanations for male-biased searching}

246 First, we tested the 'mating window hypothesis', which is that females have less incentive to 247 search if the exact timing of mating does not matter to them, so long as they acquire a mate 248 during the window. We found that the existence of a mating window does indeed favour male249 biased searching, and that this effect is compatible with both monandry and polyandry (figs. 3, 4). 
This confirms the intuitive expectation that waiting to be found becomes a sensible alternative to searching when time pressure to mate is relaxed.

Second, we tested the 'carry-over hypothesis', which is that females have less incentive to invest in searching if the costs of this investment extend beyond the period of actual searching. This hypothesis was also supported by our model (fig. 4, lower row). To appreciate why this occurs we need to consider what maintains the symmetry of male and female search effort in our baseline model (fig. 2) (see also Hammerstein and Parker 1987; Kokko and Wong 2007). In this initial scenario, if females have a longer time-out duration than males, then the operational sex ratio (OSR) becomes male-biased, and, on average, females spend little time searching. As a result, both the costs and benefits of female searching arise only during brief periods, which reduces the strength of selection on this behaviour while leaving its optimum unaffected. By contrast, if search-related mortality also arises during time-out (as posited by the carry-over hypothesis), this has a proportionately greater effect on females because of their longer time-out duration. They compensate for this by reducing their search effort. For similar reasons, Kokko et al. (2012) predicted that the sex with a longer time-out duration should have lower 'scope for competitive investment' (i.e., it should invest less in sexually selected traits, which include mate search), based on a model in which costs were expressed as a proportional decline in fitness that was independent of the amount of time spent searching.

Third, in agreement with Kokko and Wong (2007), our model supports the "sex-specific cost"

271 hypothesis, which states that females should search less if the mortality rate increase per search 272 effort is higher for females than for males (fig. 3b). 
274 We have therefore identified three alternative, non-mutually exclusive explanations for male-

275 biased searching. It will be challenging to disentangle these possibilities empirically. Our tentative 276 assessment is that the 'mating window hypothesis' is widely applicable in species with internal

277 fertilisation, where it may provide a crucial link in the causal chain between female-biased

278 parental investment and low Bateman-gradients for females (see below).

279 The 'carry-over hypothesis' is probably also relevant in many species because of the evolution of 280 morphological traits that impose costs even after searching has ended, including fitness costs 281 other than mortality (e.g., lower fecundity). It is important to note that this hypothesis does not 282 require that females are more likely to evolve such traits - only that such traits impose costs with 283 no compensating benefit for a greater proportion of a female's life (i.e. because females have a 284 longer time-out relative to time-in than do males). Finally, the 'sex-specific cost hypothesis' 285 remains plausible, but it has little a priori support and it is not clear why it would generally apply 286 across many taxa.

289 In the current models we do not find support for Kokko and Wong's (2007) prediction that 290 polyandry per se favours male-biased searching. We note that this prediction arose from a model 291 where a mating window was included to ensure polyandry, and not one designed to test directly 292 for the effect of a mating window. The two factors were therefore conflated. We found in the 293 current model that a mating window leads to male-biased searching regardless of whether or not 294 females are polyandrous (figs. 3c-d, fig. 4). In nature, of course, if mating window length correlates 295 with the degree of polyandry (which seems likely in many species), a correlation might still arise 296 between polyandry and male-biased searching. Although polyandry is associated with slightly 297 more male-biased searching than is monandry in our model, this outcome reflects one of our less 
biologically realistic assumptions: in the polyandry case we assumed that mated females continue

to behave like unmated females, so they experience costs during the time-lag with no

compensating benefit. Consequently, there is selection to reduce female search effort, for reasons

analogous to those described above for the carry-over hypothesis. We do not expect this

assumption will necessarily apply in reality because mated females might have a phenotypically

plastic response and, for example, no longer search for mates once they have acquired sperm (but see Kokko and Mappes 2013 for an alternative null hypothesis).

We have made the ad hoc assumption that mating windows are of a fixed duration. In reality, their duration might evolve. Short mating windows might be a biological constraint arising as a sideeffect of internal fertilisation (because sperm can survive in a female tract for a short while even in the absence of specialised storage organs; Orr and Brennan 2015). However, a longer mating window could evolve as a female strategy to make additional use of any pre-existing time delays before reproduction, e.g., while maturing ova, building up fat reserves, or waiting for suitable breeding conditions. By temporally decoupling mating from reproduction in this way, females can solve the problem of ensuring fertilisation before it becomes a pressing matter. Incidentally, this suggests that females differ from males in having an additional option when access to mates becomes limiting: instead of investing in mate search to increase the mate encounter rate, they might evolve earlier receptivity to increase mating window duration. This could provide yet

317 another reason why males are more likely to end up in the searching role.

\section{Conclusion}

320 In sum, our models shed new light on classic arguments about sex role evolution, by exposing the 321 logic of a mechanism that links parental investment to selection on mate search. Certain forms of 
parental investment (e.g., formation of nutrient-rich eggs; building up reserves for pregnancy) take time that can simultaneously be used as a mating window to acquire opposite sex gametes.

The sex that provides this greater investment may, in part, be released from the time pressure to mate. In principle, there could be a male mating window (e.g. males could acquire eggs before they are ready to fertilize and/or care for them), but this seems implausible in most species given the risk of lost paternity due to sperm competition. The only possible exceptions are species such as seahorses where females transfer eggs to an internal male 'compartment'. Future work could profitably explore how mating windows initially evolve, what selects for variation in their duration, and how they affect other aspects of sex role evolution, such as choosiness and parental care.

\section{Acknowledgements}

333 This research was supported by the Academy of Finland (LF, HK) and the Australian Research 334 Council (MJ, HK).

\section{Appendix}

The model by Kokko and Wong (2007) (henceforth KW) on the evolution of mate-searching

337 contained several unfortunate errors. First, they did not take into account the stable state

338 frequencies (the right eigenvector of the transition matrix; Caswell 1978; Pen and Weissing 2000)

339 when calculating selection gradients. Consequently, KW did not take into account how the 340 duration of a trait's expression (e.g. if expressed only while searching rather than throughout life)

341 affects its exposure to selection. This created a false impression that selection for searching is 342 equally strong in both sexes, leading to symmetrical evolutionary trajectories (fig. 2 in KW) even 343 when the sexes differed greatly in the time spent searching. To appreciate the problem intuitively, 
notice that selection must become infinitely weak as we approach the limiting case where one sex spends zero time searching, so that it's search effort is never 'seen' by selection.

Second, KW's equation 3c violates the so-called 'Fisher condition', because it does not evaluate to unity. The 'Fisher condition' states that fitness, hence reproductive value at birth, must be equal between the sexes when the primary sex ratio is equal (Houston and McNamara 2005). In KW's model, reproductive value in time-in is equivalent to reproductive value at birth, because there is no senescence; hence $v_{M I}^{*}=v_{F I}^{*}$ must hold to satisfy the Fisher condition.

Third, KW did not derive the equilibrium fecundity $g$ that keeps population size stable. Instead they used the arbitrary value of $g=2$ in their numerical examples. This conflicted with their assumption of constant population size when calculating the operational sex ratio. When combined, these issues lead to qualitatively different evolutionary trajectories and ESSs, as is evident by comparison of fig. 2 in KW and fig. 2 in the present paper.

To prevent perpetuation of these errors in the literature, an editorial decision was made to retract KW's paper. We share the goal of alerting readers to past errors, but - as with many models later seen as inadequate - we consider KW's contribution nevertheless a valuable one, as it provided the conceptual basis of the present work.

\section{Literature Cited}

Andersson, M. 1994. Sexual Selection. Princeton Univ. Press, Princeton. Diego. 
Clutton-Brock, T. H., and G. A. Parker. 1992. Potential reproductive rates and the operation of sexual selection. Q. Rev. Biol. 67:437-456.

Clutton-Brock, T., and A. C. J. Vincent. 1991. Sexual selection and the potential reproductive rates of males and females. Nature 351:58-60.

Hammerstein, P., and G. A. Parker. 1987. Sexual selection: games between the sexes. Pp. 119-142 in J. W. Bradbury and B. Anderson, eds. Sexual selection: testing the alternatives. Wiley, Chichester, UK. Hardling, R., H. Kokko, and K. E. Arnold. 2003. Dynamics of the caring family. Am. Nat. 161:395-412. Houston, A. I., and J. M. McNamara. 2005. John Maynard Smith and the importance of consistency in evolutionary game theory. Biol. Philos. 20:933-950.

Kokko, H., H. Klug, and M. D. Jennions. 2012. Unifying cornerstones of sexual selection: operational sex ratio, Bateman gradient and the scope for competitive investment. Ecol. Lett. 15:1340-51.

Kokko, H., and J. Mappes. 2013. Multiple mating by females is a natural outcome of a null model of mate encounters. Entomol. Exp. Appl. 146:26-37.

Kokko, H., and P. Monaghan. 2001. Predicting the direction of sexual selection. Ecol. Lett. 4:159-165. Kokko, H., and B. B. M. Wong. 2007. What determines sex roles in mate searching? Evolution (N. Y). 61:1162-1175.

McElreath, R., and R. Boyd. 2007. Mathematical models of social evolution: a guide for the perplexed. University of Chicago Press, Chicago.

Orr, T. J., and P. L. R. Brennan. 2015. Sperm storage: distinguishing selective processes and evaluating criteria. Trends Ecol. Evol. 30:261-272. Elsevier Ltd.

Pen, I., and F. J. Weissing. 2000. Towards a unified theory of cooperative breeding: the role of ecology and life history re-examined. Proc. R. Soc. B 267:2411-8.

Simmons, L. W. 2001. Sperm Competition and its Evolutionary Consequences in the Insects. Princeton University Press, Princeton.

Trivers, R. L. 1972. Parental investment and sexual selection. Pp. 1871-1971 in B. Campbell, ed. Sexual selection and the descent of man. Aldine, Chicago. 


\section{Figure legends}

Figure 1: Schematic representation of life-cycles. A. Every mating causes an immediate transition to 'time-out' (shaded area), where the consequences are processed. This applies to both sexes in the basic model, and also to males in the mating window model. B. In the monandry case of the mating window model, females experience an additional time-lag, during which they are unavailable for mating. C. In the polyandry case of the mating window model, females also experience such a time-lag, during which they are still available for mating.

\section{Figure 2: Basic model. Arrows indicate evolutionary trajectories (assuming traits evolve at rates} proportional to sex-specific selection gradients) whose end points (ESSs) are marked as black dots. Dashed lines indicate equal searching by both sexes. In the first panel, male-only searching and female-only searching are alternative ESSs. Higher search costs (in terms of $c$ ) lead to equal searching by both sexes (second row, compared to first row). Higher mate encounter rates (in terms of $M$ ) make male searching evolve faster than female searching, leading to almost vertical trajectories (third row). Other settings: $T=1, \widetilde{T}=0.01, \mu_{O}=\tilde{\mu}_{O}=0.1$.

Figure 3: Evolutionary trajectories in model versions that lead to male-biased searching. a) Basic model with carry-over costs: in addition to search-related mortality during time-in, $10 \%$ of these costs apply in time-out: $\mu_{I}[x]=0.1\left(1+x^{1.2}\right)$ and $\mu_{O}[x]=0.1\left(1+0.1 x^{1.2}\right)$ for females, versus $\tilde{\mu}_{I}[y]=0.1\left(1+y^{1.2}\right)$ and $\tilde{\mu}_{O}[x]=0.1\left(1+0.1 y^{1.2}\right)$ for males. b) Basic model with asymmetric costs: females incur $10 \%$ higher search-related mortality during time-in than males: $\mu_{I}[x]=$ $\left.0.1\left(1+1.1 x^{1.2}\right) ; \tilde{\mu}_{I}[y]=0.1\left(1+y^{1.2}\right) . c\right)$ Mating window model with monandry. d) Mating window model with polyandry. Settings in c) and d): $F=1, \widetilde{T}=T=0.01$. Default settings: $M=$ $1, \mu_{I}[x]=0.1\left(1+x^{1.2}\right), \tilde{\mu}_{I}[y]=0.1\left(1+y^{1.2}\right), \mu_{O}=\tilde{\mu}_{O}=0.1, T=1, \tilde{T}=0.01$. Search efforts 
421 are multiplicative, $f=x y$.

422

423 Figure 4: ESS search effort of males ( $y$; dash-dotted) and either monandrous or polyandrous

424 females, in the model with a mating window. As the mate-encounter coefficient $M$ increases,

425 females (but not males) search less, especially if the mating window duration $F$ is long. Other

426 settings: $\widetilde{T}=T=0.01, \mu_{O}=\tilde{\mu}_{O}=0.1, c=1.2$. Search efforts are multiplicative, $f=x y$. 